PROCEEDINGS OF THE

AMERICAN MATHEMATICAL SOCIETY

Volume 88, Number 1, May 1983

\title{
RATIONAL AUTOMORPHISMS OF GRASSMANN MANIFOLDS
}

\author{
STEPHEN BREWSTER AND WILLIAM HOMER
}

\begin{abstract}
The homotopy class of a self map of a complex projective space is well known to be classified by a degree detected in two dimensional cohomology. An analogous result is proved for the rationalization of the Grassmann manifold of complex $n$-planes in complex $N$-space, provided $N \neq 2 n$ and the degree is not zero.
\end{abstract}

Let $G_{n}\left(\mathbf{C}^{n+k}\right)$ denote the complex Grassmann manifold $U(n+k) /(U(n) \times U(k))$, and let $R$ be a subring of the rational numbers $\mathbf{Q}$ containing the integer $\mathbf{Z}$. By the grading endomorphism $\varphi$ of degree $\lambda \in R$ we mean the endomorphism defined by $\varphi(x)=\lambda^{m} x$ for $x \in H^{2 m}\left(G_{n}\left(\mathbf{C}^{n+k}\right) ; R\right)$. It is an automorphism if $\lambda$ is invertible in $R$.

THEOREM. If $\varphi$ is an endomorphism of the graded ring $H^{*}\left(G_{n}\left(\mathbf{C}^{n+k}\right) ; R\right), n \neq k$, and $\varphi$ is not identically zero on $H^{2}\left(G_{n}\left(\mathbf{C}^{n+k}\right) ; R\right)$, then $\varphi$ is a grading endomorphism.

REMARKS. 1. Since $H^{2}\left(G_{n}\left(\mathbf{C}^{n+k}\right) ; R\right) \approx R$, the degree $\lambda$ of $\varphi$ is of course determined by $\varphi(x)=\lambda x$ for any choice of $x \neq 0$ in $H^{2}\left(G_{n}\left(\mathbf{C}^{n+k}\right) ; R\right)$ (for example, $x=$ the first Chern class of the canonical $\mathbf{C}^{n}$-bundle).

2. If $\varphi$ is zero on $H^{2}\left(G_{n}\left(\mathbf{C}^{n+k}\right) ; R\right)$, we conjecture that $\varphi$ is in fact the zero endomorphism. This is proved in [GH1] when $k \geqslant 2 n^{2}-n-1$ and has been checked for a few other cases involving small values of $n$ and $k$.

3. When $n=k$, swapping the first $n$ coordinates of $\mathbf{C}^{2 n}$ with the last $n$ coordinates induces an involution $f$ of $G_{n}\left(\mathbf{C}^{2 n}\right)$. Any endomorphism which is nonzero on $H^{2}\left(G_{n}\left(\mathbf{C}^{2 n}\right)\right)$ is either a grading endomorphism or the composition of a grading endomorphism with $f^{*}$. (Note that $-f^{*}=$ id if $n=k=1$.) A proof may be found in [Hf], where the approach of [Br] is combined with some additional structure of the ring $H^{*}\left(G_{n}\left(\mathbf{C}^{n+k}\right) ; R\right)$. See Remark 9 for more details.

4. The previous remarks and our theorem may be restated as follows. Every endomorphism which is not zero on $H^{2}\left(G_{n}\left(\mathbf{C}^{n+k}\right) ; R\right)$ is the composition of a grading endomorphism with an endomorphism induced from an element of the Weyl group of $U(n) \times U(k) \subset U(n+k)$. The conjecture in Remark 2 is that one can omit the phrase "on $H^{2}\left(G_{n}\left(\mathbf{C}^{n+k}\right) ; R\right)$." The analogous statement for more general homogeneous spaces of maximal rank is not true, since there is (at least) one additional type of endomorphism (cf. [GH2]).

Received by the editors July 20, 1982.

1980 Mathematics Subject Classification. Primary 55S37; Secondary 55P62, 57T15.

Key words and phrases. Automorphisms, degree, Grassmann manifold, homogeneous space, rational homotopy, self map. 
From [GH2] we know that endomorphisms of the rational cohomology ring of $G_{n}\left(\mathbf{C}^{n+k}\right)$ are in one-to-one correspondence with homotopy classes of self maps of the rationalization of $G_{n}\left(\mathbf{C}^{n+k}\right)$. Hence our present theorem has the following geometric corollary.

COROLLARY. When $n \neq k$, the homotopy classes of self maps of the rationalization of $G_{n}\left(\mathbf{C}^{n+k}\right)$ which are nonzero on two dimensional cohomology form a group under composition which is isomorphic to the nonzero rational numbers under multiplication.

REMARKS. 5. The corollary says in other words that the group of rational automorphisms of $G_{n}\left(\mathbf{C}^{n+k}\right)$ is as small as possible. The manifold thus has a kind of "rigidity" which not surprisingly shows up in other ways as well. For applications of our theorem to group actions, see [Ba and $\mathbf{L}]$; to fixed points and coincidences of maps. [GH1 and Hf]; and to triviality of the genus set, [GM].

6. As noted above, if $k \geqslant 2 n^{2}-n-1$ we can omit the phrase "on two dimensional cohomology".

7. For a degree to be realized by a self map of $G_{n}\left(\mathbf{C}^{n+k}\right)$ itself (rather than of the rationalization), it must of course be an integer. One knows from general principles (cf. [GH2]) that infinitely many integers are so realized, but it is a difficult obstruction theory problem to determine precisely which integers these are (cf. [F] and, for some related results, $[\mathbf{H}])$.

Proof of THE TheOREM. Let $c_{i}$ denote the $i$ th Chern class of the canonical $\mathbf{C}^{n}$-bundle over $G_{n}\left(\mathbf{C}^{n+k}\right)$. If $\varphi$ is an endomorphism of $H^{*}\left(G_{n}\left(\mathbf{C}^{n+k}\right) ; R\right)$, define $a_{i}$ to be the coefficient of $c_{i}$ in $\varphi\left(c_{i}\right)$. Since the ring is free through dimension $2 k$ and we may assume, without loss of generality, that $k \geqslant n$, the $a_{i}$ 's are well defined. The main result of $[\mathrm{Br}]$ states that if all $a_{i} \neq 0$, then $\varphi$ is the grading endomorphism of degree $a_{1}$. Although the proof is written for $R=\mathbf{Z}$ it works as well for any larger subring of $\mathbf{Q}$. It now remains to show that $a_{1} \neq 0$ implies that $a_{i} \neq 0$ for all $1 \leqslant i \leqslant n$.

Since $G_{n}\left(\mathbf{C}^{n+k}\right)$ is a Kähler manifold, the 2-form representing the Kähler metric has maximal cup length. It follows that $c_{1}^{n k}=M x$, where $x$ is dual to the fundamental homology class and $M \neq 0$ is an integer. Applying $\varphi$ yields $0 \neq a_{1}^{n k} c_{1}^{n k}=M \varphi(x)$, and so $\varphi(x) \neq 0$. Given any nonzero class $y \in H^{2 m}\left(G_{n}\left(\mathbf{C}^{n+k}\right) ; R\right)$, by Poincaré duality there is a $z \in H^{2 n k-2 m}\left(G_{n}\left(\mathbf{C}^{n+k}\right) ; R\right)$ such that $y \cup z$ is a nonzero multiple of $x$. Hence $0 \neq \varphi(y \cup z)=\varphi(y) \cup \varphi(z)$, and so $\varphi(y) \neq 0$. Thus $\varphi$ is injective, so $\varphi \otimes \mathbf{Q}$ is an isomorphism and induces an isomorphism on the quotient algebra of irreducibles. Since $c_{i}$ represents the single class of irreducibles in dimension $2 i$, it follows as desired that $a_{i} \neq 0$ for $1 \leqslant i \leqslant n$.

REMARKS. 8. The proof of the main result of $[\mathrm{Br}]$ uses the following approach. The cohomology ring $H^{*}\left(G_{n}\left(\mathbf{C}^{n+k}\right) ; R\right)$ is generated by the Chern classes $c_{1}, \ldots, c_{n}$ and its ideal of relators is generated by elements $R_{1+k}, \ldots, R_{n+k}$ with $R_{i}$ in dimension $2 i$ (cf. [GH1]). Since $\varphi$ is assumed to preserve dimension, we may write $\varphi\left(c_{i}\right)=\Sigma a_{x} x$ where $x$ runs through all monomials in the $c_{i}$ 's of dimension $2 i$ and $a_{x} \in R$. The requirement that $\varphi\left(R_{1+k}\right)$ must be a multiple of $R_{1+k}$ restricts the possibilities for the coefficients $a_{x}$. In [GH1] it was shown that when $k \geqslant 2 n^{2}-n-1$, this 
restriction already requires $\varphi$ to be a grading endomorphism. To treat the remaining cases in $[\mathbf{B r}]$, it was necessary to consider the restrictions on the $a_{x}$ resulting from the additional requirements that $\varphi\left(R_{i}\right)$ should be in the ideal generated by $R_{1+k}, \ldots, R_{i}$, where $1+k<i \leqslant n+k$. The restrictions which come from these higher dimensions are less convenient than those used in [GH1], and the proof is long and very computational. The coefficients $a_{x}$ are, for the most part, considered in a lexicographical order on their subscripts.

9. The innovation in [Hf] is first to show, via the "hard Lefschetz theorem," that $a_{i}=\varepsilon_{i} a_{1}^{i}$, where $\varepsilon_{i} \in\{1,-1\}$ and the coefficient of $c_{i}$ in $\varphi\left(c_{i}\right)$ is denoted by $a_{i}$ (rather than by $a_{c_{i}}$ as in Remark 8). Given this preliminary information, a substantially shortened version of the argument given in [Br] suffices to complete the proof.

ACKNOWLEDGMENTS. We would like to thank Guido Mislin for the suggestion that led to the proof of our theorem, Henry Glover for a number of helpful discussions, and Michael Hoffman for providing us with a copy of his dissertation.

\section{REFERENCES}

[Ba] Allen Back, Homotopy rigidity for Grassmannians, Proc. Amer. Math. Soc. 80 (1980), 327-332.

[Br] Stephen Brewster, Automorphisms of the cohomology ring of finite Grassmann manifolds, Ph.D. dissertation, Ohio State University, 1978.

[F] Eric Friedlander, Maps between localized homogeneous spaces, Topology 16 (1977), 205-216.

[GH1] Henry Glover and William Homer, Endomorphisms of the cohomology ring of finite Grassmann manifolds (Proc. Northwestern Univ. Conf. Geometric Applications of Homotopy Theory), Lecture Notes in Math., vol. 657, Springer-Verlag, Berlin and New York, 1978, pp. 170-193.

[GH2] __ Self maps of flag manifolds, Trans. Amer. Math. Soc. 267 (1978), 423-434.

[GM] Henry Glover and Guido Mislin, Genus of generalized flag manifolds, Enseign. Math. (2) 27 (1981), 211-219.

[Hf] Michael Hoffman, Cohomology endomorphisms of complex flag manifolds, Ph.D. dissertation, Massachusetts Institute of Technology, 1981.

[H] William Homer, Nontrivial splittings of trivial bundles on projective space, preprint.

[L] Arunas Liulevicius, Equivariant K-theory and homotopy rigidity, Topology Symposium, Siegen 1979, Lecture Notes in Math., vol. 788, Springer-Verlag, Berlin and New York, pp. 340-358.

Department of Mathematics, Central State University, Wilberforce, Ohio 45384

Department of Mathematics and Statistics, Memorial University of Newfoundland, St. JoHN's, NeWfoundLAND, A1B 3X7, CANADA 